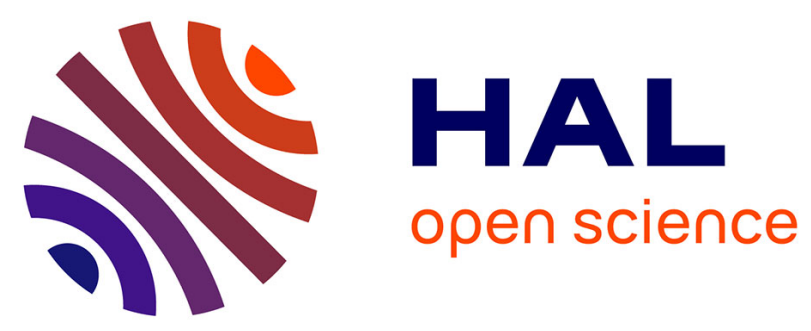

\title{
Multiple-Scattering Extended X-Ray Absorption Fine Structure Analysis of Spinel Ferrites: Cation Site Distribution in NiZn-Ferrite Films
}

\author{
V. Harris, C. Williams, M. Abe, Q. Zhang
}

\section{- To cite this version:}

V. Harris, C. Williams, M. Abe, Q. Zhang. Multiple-Scattering Extended X-Ray Absorption Fine Structure Analysis of Spinel Ferrites: Cation Site Distribution in NiZn-Ferrite Films. Journal de Physique IV Proceedings, 1997, 07 (C1), pp.C1-215-C1-218. 10.1051/jp4:1997181 。 jpa-00255128

HAL Id: jpa-00255128

https://hal.science/jpa-00255128

Submitted on 1 Jan 1997

HAL is a multi-disciplinary open access archive for the deposit and dissemination of scientific research documents, whether they are published or not. The documents may come from teaching and research institutions in France or abroad, or from public or private research centers.
L'archive ouverte pluridisciplinaire HAL, est destinée au dépôt et à la diffusion de documents scientifiques de niveau recherche, publiés ou non, émanant des établissements d'enseignement et de recherche français ou étrangers, des laboratoires publics ou privés. 


\title{
Multiple-Scattering Extended X-Ray Absorption Fine Structure Analysis of Spinel Ferrites: Cation Site Distribution in NiZn-Ferrite Films
}

\author{
V.G. Harris, C.M. Williams*, M. Abe** and Q. Zhang** \\ U.S. Naval Research Laboratory, Washington, D.C. 20375-5000, U.S.A. \\ * Morgan State University, Baltimore, MD 21339, U.S.A. \\ ** Tokyo Institute of Technology, Okayama, Meguro-Ku, Tokyo 152, Japan
}

\begin{abstract}
We have applied extended X-ray absorption fine structure (EXAFS) spectroscopy to study the cation distribution in a series of spin-sprayed NiZn-ferrite films. A least-squares fitting of experimental EXAFS data with theoretical, multiple-scattering, EXAFS data allowed the quantitative determination of site distributions for all transition metal cations.
\end{abstract}

\section{INTRODUCTION}

In recent years there has been a resurgence in the study of spinel ferrites largely fueled by the need for high-quality, lowloss, films for the development of magnetic microwave integrated-circuit devices. Because samples grown via vaporquenching techniques are typically not at equilibrium, trends derived from experiments on bulk samples cannot be readily extrapolated to thin films. As a result, the careful characterization of thin film ferrites is essential. In spinel ferrites it is the distribution of the cation species on the octahedral and tetrahedral sites which most influences the materials' magnetic and electronic properties. Probes that can provide this information include X-ray diffraction (XRD) [1] and neutron diffraction (ND) [2], and Mossbauer effect (ME) [3]. However, techniques which are useful for the characterization of bulk materials are often of limited use when it comes to thin films. For example, when XRD and ND are applied to thin films the small mass and crystalline texture of the samples severely limit their usefulness. While ME has evolved to be a mainstay technique for the study of the environment of Fe ions, often being able to provide both structural and magnetic information for bulk and thin film samples, it is less effective when the focus is on dilute or non-magnetic additives. The development of novel thin film ferrites necessitates the need for improved techniques to study the local structure and chemistry of thin films with emphasis on cation site distribution.

In this paper we describe the use of conversion-electron, extended X-ray absorption fine structure (EXAFS) as a tool to study the site distribution of cations in ferrite films. Because this technique offers element specificity, as well as local structure and chemical sensitivity, it is ideally suited for such a study. Furthermore, this technique is readily applied to thin films on thin supporting substrates without the need for additional sample preparation.

\section{EXPERIMENTAL}

Samples studied here are spin-sprayed [4] NiZn-ferrite films $(t=0.5 \mu \mathrm{m})$ where the Ni concentration is fixed and the $\mathrm{Zn}$ concentration is varied at the expense of the Fe. The films were deposited onto glass substrates with compositions: $\mathrm{Ni}_{0} .15 \mathrm{Zn}_{y} \mathrm{Fe}_{2.85-y \mathrm{O}_{4}}(\mathrm{y}=0.16,0.23,0.40,0.60)$. Magnetization measurements were performed using a vibrating sample magnetometer and the results are correlated with the site distribution results obtained from the EXAFS measurements. Powder samples of $\mathrm{ZnFe}_{2} \mathrm{O}_{4}, \mathrm{NiFe}_{2} \mathrm{O}_{4}$, and $\mathrm{Fe}_{3} \mathrm{O}_{4}$ were studied as empirical standards for use in the EXAFS modeling analysis.

Hastings and Corliss [5] found $\mathrm{ZnFe}_{2} \mathrm{O}_{4}$ to be a normal spinel, meaning that the 8 divalent ions occupy the 8 tetrahedral (A) sites and the 16 trivalent ions occupy the 16 octahedral (B) sites, and $\mathrm{NiFe}_{2} \mathrm{O}_{4}$ to be an inverse spinel, meaning that the 8 divalent ions occupy 8 of the $16 \mathrm{~B}$-sites and the 16 trivalent ions randomly occupy the remaining sites. In studies of bulk powders it was found that when $\mathrm{Zn}$ is added to the $\mathrm{NiFe}_{2} \mathrm{O}_{4}$ at the expense of $\mathrm{Ni}$, the $\mathrm{Zn}$ ions preferentially occupy the A-sites, displacing some of the Fe ions onto the B-sites [5]. For our films one might expect that when $\mathrm{Zn}$ is added at the expense of the $\mathrm{Fe}$ ions a similar redistribution takes place, namely, $\mathrm{Zn}$ ions preferentially occupy the A-sites and displace a larger fraction of $\mathrm{Fe}$ ions onto the $\mathrm{B}$-sites. The $\mathrm{Ni}$ ions are expected to reside on the $\mathrm{B}$-sites for all samples. As will be discussed, the cation distribution is not that simple.

EXAFS data collection was performed using the total electron-yield (TEY) detection scheme [6, 7] at the National Synchrotron Light Source (Brookhaven National Laboratory, Upton, NY, USA) and entailed measuring the X-ray absorption 
coefficient encompassing the $\mathrm{K}$ absorption edges of $\mathrm{Fe}(7111 \mathrm{eV}), \mathrm{Ni}(8333 \mathrm{eV})$ and $\mathrm{Zn}(9659 \mathrm{eV}){ }^{1}$ Following established EXAFS analysis procedures [9] the data were Fourier transformed to radial coordinates, i.e. EXAFS radial distribution functions (E-RDFs). In this form the data directly reflect the average local environment around the absorbing atoms where the peaks in the transform typically represent shells of atoms around the absorber [9] $]^{2}$. Data collected from standards of $\mathrm{Fe}_{3} \mathrm{O}_{4}$, $\mathrm{ZnFe}_{2} \mathrm{O}_{4}$, and $\mathrm{NiFe}_{2} \mathrm{O}_{4}$ were similarly analyzed. In addition, theoretical EXAFS data, generated using the multiple-scattering FEFF (v.6.01) codes $[10,11]$, were similarly analyzed and used in least-squares fitting the unknown EXAFS data to determine the fractional site occupancy of the absorbing cations. Henceforth, data generated from these codes will be referred to as FEFF data.

\section{RESULTS AND DISCUSSION}

The spinel ferrite unit cell has the $f d 3 m$ space group and is based on a close-packed oxygen lattice where metal cations reside on 8 of the 64 tetrahedral sites and 16 of the 32 octahedral sites. The $O$ ions reside on an fcc lattice of $a_{0} / 2$, while the A-site ions reside on the interstices of 2 interpenetrating fcc lattices of $a_{0}$, and the B-site ions reside on the interstices of 4 interpenetrating fec lattices of $a_{0}$. Figure $1(a)$ illustrates two octets of a single spinel ferrite unit cell illustrating the A- and Bsite locations relative to each other and to the $O$ lattice. The A-site cations have $4 \mathrm{O}$ neighbors at a distance of $\approx 1.9 \AA$, while the B-site cations have $6 \mathrm{O}$ neighbors at a distance of $\approx 2.1 \AA[12,13]$.

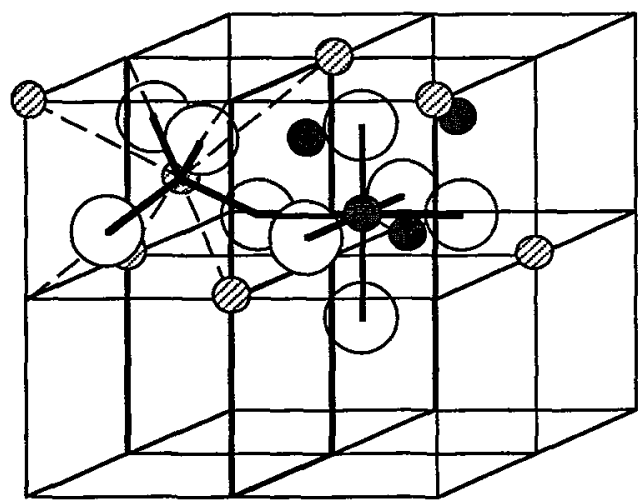

after E.W. Gorter, Philips Res. Rep., 9, 321 (1954).

(a)

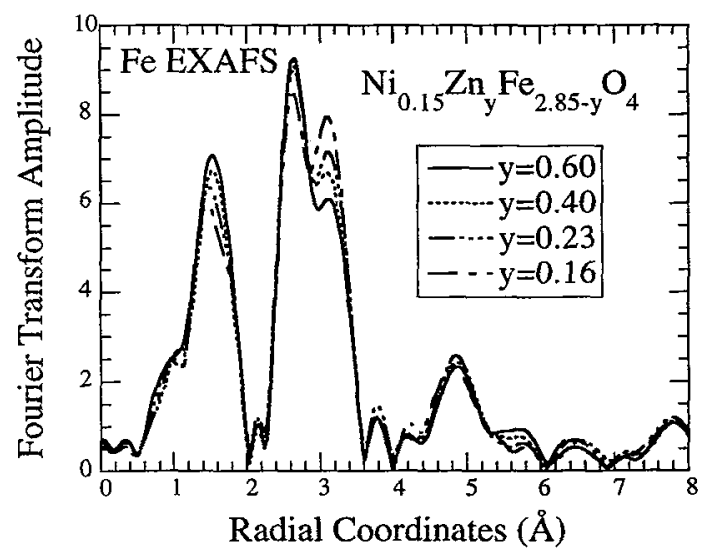

(b)

Figure 1: (a) Two octets of a single spinel ferrite unit cell (type: $\mathrm{AB}_{2} \mathrm{O}_{4}$ ) illustrating the octahedral (solid circles) and tetrahedral (hashed circles) symmetry and coordination. (b) Fourier transformed Fe EXAFS data acquired from the $\mathrm{Ni}_{0.15} \mathrm{Zn}_{\mathrm{y}} \mathrm{Fe}_{2.85} \mathrm{y} \mathrm{O}_{4}$ film samples. All transforms were obtained using $k^{3}$-weighting and a $k$-range of $2.5-12.5 \AA^{-1}$.

Figure 1(b) is a plot of the Fourier transformed Fe EXAFS collected from the four $\mathrm{Ni}_{0.15} \mathrm{Zn}_{\mathrm{y}} \mathrm{Fe}_{2.85} \mathrm{y} \mathrm{O}_{4}$ samples. These data have all been analyzed using identical parameters and plotted on the same $\mathrm{x}$ - and $\mathrm{y}$-axes to allow direct comparisons between sample sets. By comparing the Fourier transformed $\mathrm{Fe}$ EXAFS data with the data collected from standards of $\mathrm{Fe}_{3} \mathrm{O}_{4}$, $\mathrm{NiFe}_{2} \mathrm{O}_{4}$, and $\mathrm{ZnFe}_{2} \mathrm{O}_{4}$ it is clear that these profiles illustrate features characteristic of both A- and B-site environments [12, 13]. It is also clear, from Fig. 1(b), that the amplitudes of these Fourier features vary from sample to sample in a systematic manner. Upon close inspection, one sees that the variations are largest in the first three peaks of the Fourier transforms. The large asymmetric peak, centered near $1.5 \AA$, corresponds with the two $\mathrm{Fe}-\mathrm{O}$ bond distances corresponding to the $\mathrm{Fe}$ ions on the A- and B-sites.[12, 13] The large split peak feature, centered near $2.9 \AA$, corresponds with several different atom pair correlations as well as multiple-scattering (MS) contributions.[13] The low-r peak on this split feature (near $2.7 \AA$ ) includes contributions only from the $\mathrm{Fe}_{\mathrm{B}}-\mathrm{M}_{\mathrm{B}}$ (M; metal cation) correlations. In contrast, the high-r peak on this split feature (near 3.1 $\AA$ ) includes contributions from $\mathrm{Fe}_{\mathrm{A}}-\mathrm{M}_{\mathrm{A}}, \mathrm{Fe}_{\mathrm{A}}-\mathrm{O}, \mathrm{Fe}_{\mathrm{A}}-\mathrm{M}_{\mathrm{B}}$, and $\mathrm{Fe}-\mathrm{O}$ correlations: About $12 \%$ of this peak's amplitude arises from MS contributions. In Fig. 1(b) the high- $r$ peak increases in amplitude with decreasing $\mathrm{Zn} / \mathrm{Fe}$ while the low- $r$ peak shows the opposite trend. The peak centered near $1.5 \AA$ decreases in amplitude with decreasing $\mathrm{Zn} / \mathrm{Fe}$. All of these trends can be qualitatively explained by the site distribution of the $\mathrm{Zn}$ and Fe cations with increasing $\mathrm{Zn} / \mathrm{Fe}$. When $\mathrm{Zn}$ is added to the sample it preferentially occupies the A-sites forcing a larger fraction of Fe ions to occupy the B-sites. The trends in the splitpeak feature near $2.9 \AA$ reflect the changes in $\mathrm{Fe}_{\mathrm{B}} / \mathrm{Fe}_{\mathrm{A}}$ as a function of $\mathrm{Zn} / \mathrm{Fe}$. The oxygen peak varies in amplitude due to the

\footnotetext{
${ }^{1}$ The NRL beamline, X23B, was used for data collection. The X-ray and optical properties of this beamline are presented in Ref. [8]

${ }^{2}$ Because electron phase shift corrections have not been included at this stage in the analysis the peak positions of the Fourier transformed EXAFS data do not correlate directly with bond distances but are instead shifted to lower radial distances by an amount equal to the unique electron phase shift of the pair correlations contributing to the Fourier peak.
} 
change in occupancy between the 6-fold coordination B-site and the 4-fold coordination A-site. Although this type of qualitative evaluation can be useful, a quantitative treatment which provides the fractional occupancy of cations on A- and Bsites would undoubtedly provide a more comprehensive understanding of this system.

The traditional approach to quantitative EXAFS analysis entails the least-squares fitting of a fixed range of Fourierfiltered (FF) EXAFS data with parameterized single-scattering simulated EXAFS data [10]. However, in this case the MS contributions to the high-r peak at $3.1 \AA$ of Fig. 1(b) preclude this approach. To circumvent this limitation we have generated data using the FEFF v.6.01 codes[11] for the A- and B-site environments which include the MS contributions. The fitting analysis then followed the traditional approach, namely, of Fourier-filtering an r-space range extending from 2 to $4 \AA$, and least-squares fitting the phase and amplitude of these data with similarly analyzed FEFF data in photoelectron wave vector space. [Henceforth, these data will be referred to as FF EXAFS and FF FEFF data, respectively.]
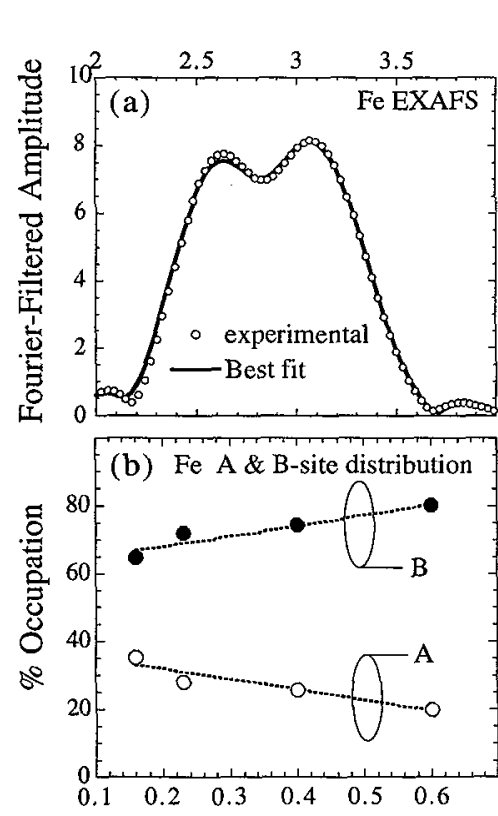

Radial Coordinates $(\AA)$
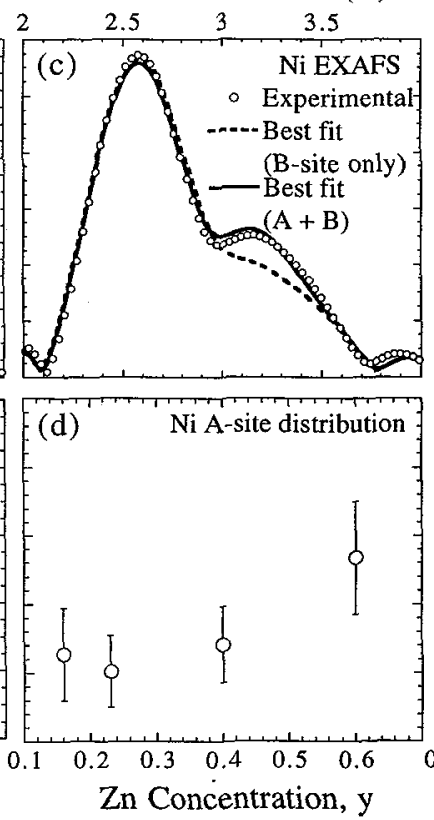

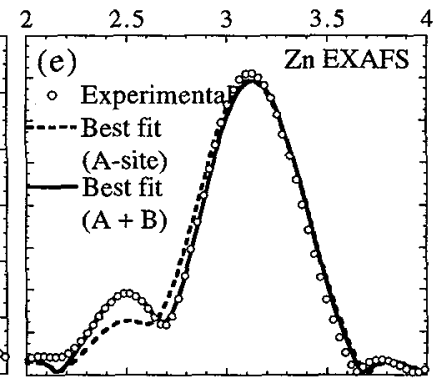

(f) $\quad \mathrm{Zn}$ B-site distribution

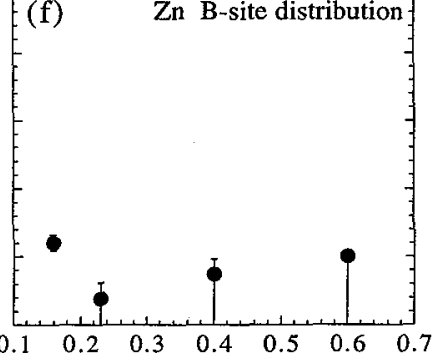

Figures 2 (a.f): Panels (a, c, and e) are Fourier-filtered $\mathrm{Fe}, \mathrm{Ni}$ and $\mathrm{Zn}$ EXAFS data, respectively, for the $\mathrm{Ni}_{0.15} \mathrm{Zn}_{0.16} \mathrm{Fe}_{2.69} \mathrm{O}_{4}$ sample with best fits determined by least-squares fitting the data with FEFF data. Panels (c) and (e) illustrate the need for a combination of A- and B-site components to adequately fit the entire data range. Panels (b), (d), and (f) show the results of the fitting procedures for all samples as the site occupation of (b) Fe A- and B-site cations, (d) Ni A-site cations, and (f) Zn B-site cations, as a function of $\mathrm{Zn}$ concentration $(\mathrm{y})$.

Prior to fitting the unknown FF EXAFS data, the FF FEFF data were fit to the standards' data to allow a refinement of the Debye-Waller ordering parameters and the $v_{0}$ correction. ${ }^{3}$ The results of fitting the standards were consistent with the results presented in ref. [5]. The A- and B-site FF FEFF data were then applied in a least-squares fit to the unknown data with the only adjustable parameter being the fractional occupancy of the A- and B-sites. These fits are presented in r-space (Fig. 2(a, c, and e)) for an improved evaluation of the differences between theory and experiment. The results of the fitting analyses are presented in Figures 2 (b, $d$, and $f$ ). The FF Fe, Ni, and $\mathrm{Zn}$ EXAFS data for the $y=0.16$ sample and the best fit determined using a combination of A- and B-site FF FEFF data are presented in Figs. 2 (a, c, and e), respectively. These data are representative of the FF EXAFS data for the other compositions and their fitted data. Although slight differences exists between the experimental and calculated data, these differences are much smaller than the differences in the E-RDFs between the different compositions, lending increased confidence to the fitting results. The error bars plotted on these symbols reflect the best fit to the mean \pm one standard deviation of the FF EXAFS data. ${ }^{4}$ Although a mixture of A- and B-site environments is expected to be needed to fit the Fe EXAFS, one would have expected from experiments performed on bulk samples that the $\mathrm{Zn}$ and Ni EXAFS would have required only $\mathrm{A}$ - and B-components, respectively. In panel (c) it is clear that the best fit

\footnotetext{
${ }^{3}$ The only adjustable parameters in fitting the standards with FEFF data were the $\theta_{D}$ and $v_{0}$ parameters. The $\theta_{D}$ parameter is used to adjust the Debye-Waller coefficients for all atomic correlations of the cluster according to the Debye model, while the v0 correction adjust for the uncertainty between the value of $\mathrm{E}_{0}$ assigned theoretically and experimentally.

4 Error bars were determined by merging several individual data scans after normalization, background removal, and conversion to photoelectron wave vector space, thus allowing the determination of a mean and standard deviation which reflects both the data collection statistics and the uncertainty introduced to the data by the background removal procedure.
} 
obtained using B-site FF FEFF alone does not fit the Ni data in the 3-3.5 $\AA$ range. However, by introducing a small fraction of A-site FF FEFF the fit improves dramatically. Indeed, Fig. 2 (d) indicates that the amount of A-site Ni cations increases with increasing $\mathrm{Zn}$ content reaching a maximum of $23 \%$ occupancy for $\mathrm{y}=0.6$. Similar to the Ni data of panel (c), the $\mathrm{Zn} F \mathrm{~F}$ EXAFS data in panel (e) is best fit using a combination of A- and B-site FF FEFF data. In the case for $y=0.16,6 \% \mathrm{~B}$-site FF FEFF is needed to fit the data range of $2.25-2.75 \AA$. Although the B-site component appears to track the Ni fitting results of panel (d), only the value for the $y=0.16$ composition is statistically significant. For the other compositions best fits of the mean - one standard deviation are equally good without a $B$ component.

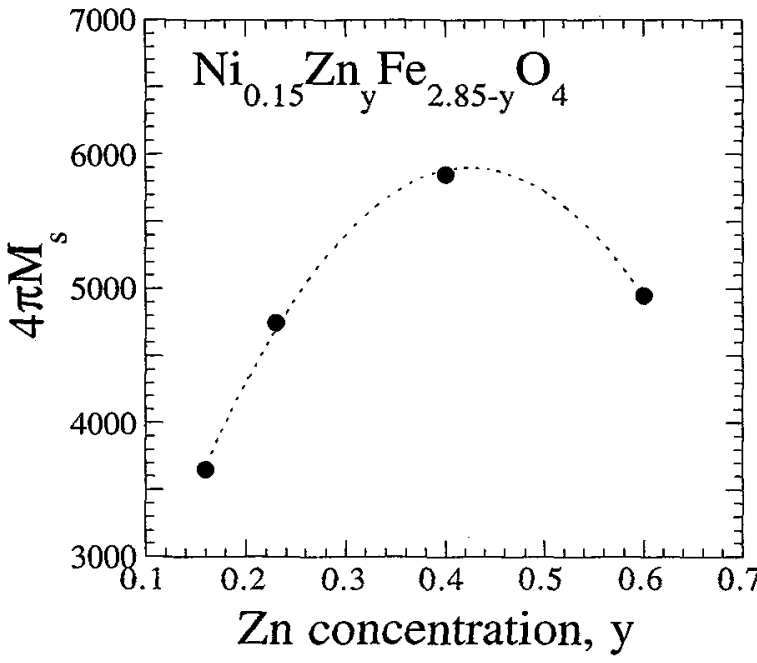

Figure 3: Saturation magnetization $(4 \pi \mathrm{Ms})$ versus $\mathrm{Zn}$ concentration.

It was mentioned earlier that Ni-ferrite has an inverted cation distribution and, consequently, its magnetization arises solely from the $\mathrm{Ni}^{+2}$ ions: the $\mathrm{A}$ and $\mathrm{B}$-site $\mathrm{Fe}$ cations align antiferromagnetically and provide no net moment. The introduction of $\mathrm{Zn}^{+2}$ ions, which prefer the A-sites, upsets the ferric balance by displacing the $\mathrm{Fe}$ cations from the $\mathrm{A}$ - to B-sites. The effect of a small amount of $\mathrm{Zn}$ is to increase the saturation magnetization with the now unbalanced $\mathrm{Fe}$ moments adding to those of the $\mathrm{Ni}^{+2}$ ions. In Fig. 3 we measure magnetization values to increase near linearly with increased $\mathrm{Zn}$ concentration for $0 \leq \mathrm{y} \approx 0.4$ and then decrease for the $y=0.6$ sample. We believe the decrease in magnetization is due to a deterioration of the superexchange between the A-B sites brought about by lattice distortions caused by the increased amount of $\mathrm{Zn}$ ions on the A-sites. We measure as evidence of this a modest $0.5 \%$ dilation of the $\mathrm{Zn}_{\mathrm{A}}-\mathrm{O}$ bond for the $y=0.6$ compared with the $0.16 \leq y \leq 0.4$ samples. However, because the Curie temperature of these samples also changes with increased $\mathrm{Zn}$ concentration, a Curie temperature effect cannot be ruled out. Low temperature magnetization studies are underway to clarify this effect.

In summary, we have performed EXAFS measurements including fitting analysis using simulated, multiple-scattering, EXAFS data on a series of NiZn-ferrite films to investigate the cation distribution on the A- and B-sites. We find that the $\mathrm{Zn}$ cations occupy only A-sites, save for one sample $(y=0.16)$ in which we measure $6 \%$ of the $\mathrm{Zn}$ ions occupying B-sites. The Fe cations are found to occupy both $A$ - and $B$-sites in a ratio strongly influenced by the $\mathrm{Zn} / \mathrm{Fe}$. We measure the $\mathrm{Ni}$ ions to occupy both $\mathrm{A}$ - and B-sites with a preference for $\mathrm{B}$ sites. The $\mathrm{Ni} \mathrm{A}$-site occupation is small for small $\mathrm{Zn}$ concentrations and increases to $\approx 25 \%$ at a maximum $\mathrm{Zn}$ concentration of $\mathrm{y}=0.6$. The distribution of $\mathrm{Ni}$ ions in these films is somewhat surprising when one considers the results of neutron studies on similar bulk systems which found Ni residing only on the B-sites. We believe the large fraction of A-site Ni ions to be intrinsic to the thin film structure that results from the non-equilibrium processing and might be characteristic of other thin film processing techniques. Because of the relatively small number of atoms contributed to the average unit cell the site distribution of $\mathrm{Ni}$ does not have a profound impact on the sample's magnetization. This represents the first use of EXAFS to determine cation site distribution in thin film ferrites. The use of EXAFS, specifically quantitative fitting analysis using multiple-scattering FEFF codes, will be instrumental in understanding the magnetic and electronic properties of novel thin film ferrites.

This research was carried out, in part, at the National Synchrotron Light Source (Brookhaven National Laboratories, Upton, NY), which is sponsored by the U.S. Department of Energy.

\section{References}

[1] A. Claasen, Proc. Phys. Soc., 38, 482, 1925-1926.

[2] C. G. Shull, E. O. Wollan, and W. C. Koehler, Phys. Rev., 84, 912, 1951.

[3] G. K. Wertheim, J. Appl. Phys, 32, 110S, 1961.

[4] M. Abe and Y. Tamaura, J. Appl. Phys., 55, 2614, 1984.

[5] J. M. Hastings and L. M. Corless, Rev. Mod. Phys., 25, 114, 1953.

[6] A. Erbil, G. S. C. III, R. Frahm, and R. F. Boehme, Phys. Rev., B37, 2450, 1988.

[7] W. T. Elam, J. P. Kirkland, R. A. Neiser, and P. D. Wolf, Phys. Rev., B38, 26, 1988.

[8] R. A. Neiser, J. P. Kirkland, W. T. Elam, and S. Sampath, Nucl. Instrm. Methods Phys. Res. Sect., A266, 220, 1988.

[9] D. E. Sayers and B. A. Bunker, in X-Ray Absorption: Principles, Applications, Techniques of EXAFS, SEXAFS, and XANES, D. C. Koningsberger and R. Prins, Eds. New York, NY: Wiley, 1988.

[10] J. J. Rehr, J. M. d. Leon, S. I. Zabinsky, and R. C. Albers, J. Amer. Chem. Soc, 113, 5135, 1991.

[11] J. J. Rehr, S. I. Zabinsky, and R. C. Albers, Phys. Rev. Lett., 69, 3397, 1992.

[12] V. G. Harris, N. C. Koon, C. M. Williams, Q. Zhang, M. Abe, J. P. Kirkland, and D. A. McKeown, IEEE Trans. on Magn., 31, 3473, 1995.

[13] V. G. Harris, N. C. Koon, C. M. Williams, Q. Zhang, M. Abe, and J. P. Kirkland, Appl. Phys. Lett., 68, 2082, 1996. 